\title{
ON SHIMURA LIFTING OF MODULAR FORMS
}

\author{
By
}

Shigeaki TsuYumINE

0. Let $k, N \in N, 4 \mid N$. Let $M_{k+1 / 2}\left(N, \chi_{0}\right)$ denote the space of modular forms for $\Gamma_{0}(N)$ of weight $k+1 / 2$ with a character $\chi_{0}(\bmod N)$. Lifting maps of cusp forms in $M_{k+1 / 2}\left(N, \chi_{0}\right)$ to modular forms of integral weight was first studied by Shimura [7] and later by Niwa [4]. The domain of the map is extended to $M_{k+1 / 2}\left(N, \chi_{0}\right)$ by van Asch [1] in case that $\chi_{0}$ is real and $N=4 p$ for $p$ prime, and by Pei [5] in case that $\chi_{0}$ is real and $N / 4$ is square-free. In the present paper we consider the lifting map without any condition on $N$ and $\chi_{0}$, and extend the domain of the map to $M_{k+1 / 2}\left(N, \chi_{0}\right)$ for $k \geq 2$.

To show the assertion, we take some specific modular forms in $M_{k+1 / 2}\left(N, \chi_{0}\right)$ which together with cusp forms, span $M_{k+1 / 2}\left(N, \chi_{0}\right)$. Further we construct their liftings explicitly. It proves our main result. It may be expected to have further application to study of special values of $L$-series of Hecke eigen cusp forms, as in Zagier [9], Kohnen-Zagier [3] where the lifting of some particular modular forms plays an important role.

1. We denote by $\boldsymbol{N}, \boldsymbol{Z}, \boldsymbol{C}$, the set of natural numbers, the ring of integers and the complex number field respectively. For a prime $p \in N, v_{p}$ denotes the $p$-adic valuation. For $N \in \boldsymbol{N},(\boldsymbol{Z} / N)^{*}$ denotes the group of Dirichlet characters $(\bmod N)$. When $N=1$, the group is consisting of a constant 1 . The identity element of $(\boldsymbol{Z} / N)^{*}$ is denoted by $1_{N}$. A group consisting of invertible elements in $\boldsymbol{Z} / N$ is denoted by $(\boldsymbol{Z} / N)^{\times}$. If $\chi \in(\boldsymbol{Z} / N)^{*}$ and $e \in N$, the $\chi^{(e)}$ denotes a character $(\bmod e N)$ obtained by $\chi^{(e)}(d)=\chi(d) \quad((d, e)=1), 0((d, e) \neq 1)$. In case that all prime factors of $e$ appear as factors of $N$, then $\chi^{(e)}$ is equal to $\chi$. For $a \in \boldsymbol{Z}$ and for an odd $b \in \boldsymbol{N},(a / b)$ denotes the Jacobi-Legendre symbol where it is 0 if $(a, b) \neq 1$. If $D$ is a discriminant of a quadratic field, then $\chi_{D}$ denote the Kronecker-Jacobi-Legendre symbol. We put $\chi_{D}=1$ for $D=1$.

Let $\mathfrak{S}$ denote the upper-half plane $\{z \in C \mid \operatorname{Im} z>0\}$. The group $S L_{2}(\boldsymbol{Z})$ acts on $\mathfrak{H}$ by the usual modular transformation sending $z \in \mathfrak{G}$ to $M z=(a z+b) /$ 
$(c z+d), M=\left(\begin{array}{ll}a & b \\ c & d\end{array}\right)$. For $N \in N$ which is not necessarily divisible by 4 , let $\Gamma_{0}(N):=\left\{\left(\begin{array}{ll}a & b \\ c & d\end{array}\right) \in S L_{2}(\boldsymbol{Z}) \mid c \equiv 0(\bmod N)\right\}, \quad \Gamma_{1}(N):=\left\{\left(\begin{array}{ll}a & b \\ c & d\end{array}\right) \in S L_{2}(\boldsymbol{Z}) \mid\right.$ $a \equiv d \equiv 1, c \equiv 0(\bmod N)\}$. Let $k \in \boldsymbol{N}$ and $\chi_{0} \in(\boldsymbol{Z} / N)^{*}$. A holomorphic function $f$ on $\mathfrak{H}$ is called a modular form for $\Gamma_{1}(N)$ of weight $k$ (resp. a modular form for $\Gamma_{0}(N)$ of weight $k$ with character $\left.\chi_{0}\right)$ if it satisfies that (i) $\left(\left.f\right|_{M}\right)(z)=f(z)$ for $M \in \Gamma_{1}(N)$ (resp. $\left(\left.f\right|_{M, \chi_{0}}\right)(z)=f(z)$ for $\left.M \in \Gamma_{0}(N)\right)$, and that (ii) $f$ is holomorphic also at cusps, where $\left(\left.f\right|_{M}\right)(z)=(c z+d)^{-k} f(M z) \quad\left(\operatorname{resp} .\left(\left.f\right|_{M, \chi_{0}}\right)(z)=\right.$ $\left.\chi_{0}(d)^{-1}(c z+d)^{-k} f(M z)\right)$ for $M=\left(\begin{array}{ll}a & b \\ c & d\end{array}\right)$. We denote by $M_{k}\left(\Gamma_{1}(N)\right)$ (resp. $\left.M_{k}\left(N, \chi_{0}\right)\right)$, the space of such modular forms, and by $S_{k}\left(\Gamma_{1}(N)\right)\left(\operatorname{resp} . S_{k}\left(N, \chi_{0}\right)\right)$, the space of such cusp forms. The orthogonal complement of $S_{k}\left(\Gamma_{1}(N)\right)$ (resp. $\left.S_{k}\left(N, \chi_{0}\right)\right)$ in $\boldsymbol{M}_{k}\left(\Gamma_{1}(N)\right)$ (resp. $\left.\boldsymbol{M}_{k}\left(N, \chi_{0}\right)\right)$ with respect to the Petersson product, is called a space of Eisenstein series, and it is denoted by $E_{k}\left(\Gamma_{1}(N)\right.$ ) (resp. $\left.E_{k}\left(N, \chi_{0}\right)\right)$. We have

$$
M_{k}\left(\Gamma_{1}(N)\right)=\bigoplus_{\chi_{0}} M_{k}\left(N, \chi_{0}\right), \quad E_{k}\left(\Gamma_{1}(N)\right)=\bigoplus_{\chi_{0}} E_{k}\left(N, \chi_{0}\right)
$$

where $\chi_{0}$ runs over $(\boldsymbol{Z} / N)^{*}$.

The number $v_{0}(N)\left(\right.$ resp. $\left.v_{1}(N)\right)$ of inequivalent cusps of $\Gamma_{0}(N)\left(\right.$ resp. $\left.\Gamma_{1}(N)\right)$ is equal to $\sum_{N_{1} N_{2}=N} \varphi\left(\left(N_{1}, N_{2}\right)\right)$ (resp. $2(N=2), 3(N=4), 1 / 2 \sum_{N_{1} N_{2}=N} \varphi\left(N_{1}\right)$. $\left.\varphi\left(N_{2}\right)(N \geqq 3, \neq 4)\right)$ where $\varphi$ denotes the Euler function and $N_{i}$ 's are positive divisors of $N$. A complete system of representatives of inequivalent cusps of $\Gamma_{0}(N)$ is given by $\left\{i / N_{2}\left|0<N_{2}, N_{2}\right| N\right.$, i's being representatives of $\left.\left(Z /\left(N_{1}, N_{2}\right)\right)^{\times}\right\}$. That of $\Gamma_{1}(N)$ is given as follows. Let $i \in\left(Z / N_{2}\right)^{\times}, j \in\left(Z / N_{1}\right)^{\times}$. Then for suitable $a, b \in \boldsymbol{Z}$, a fraction $\left(i+a N_{2}\right) /\left(\left(j+b N_{1}\right) N_{2}\right)\left(\left(j+b N_{1}, N_{2}\right)=1\right)$ is reduced, one of which we denote by $\left[i, j, N_{2} ; N\right]$. Then a complete system is $\left\{\left[i, j, N_{2} ; N\right] \mid\right.$ $\left.N_{1} N_{2}=N,(i, j) \in\left(Z / N_{2}\right)^{\times} \times\left(Z / N_{1}\right)^{\times} /\{ \pm 1\}\right\}$. By abuse of language, the rational numbers sometimes mean cusps on the corresponding modular curve.

LEMMA 1. (1) Via the canonical map of the modular curve of $\Gamma_{1}(N)$ onto that of $\Gamma_{0}(N)$, a cusp $\left[i, j, N_{2} ; N\right]$ is mapped to a cusp $i j^{-1} / N_{2}$ where $j^{-1}$ is denoting an inverse of $j\left(\bmod N_{1}\right)$.

(2) Let $N=N_{1} N_{2}$ and let $N_{2}|M, M| N$. If $\rho$ denotes the natural surjective map of the modular curve of $\Gamma_{1}(N)$ onto that of $\Gamma_{1}(M)$, then $\rho^{-1}\left(\left[i, j, N_{2} ; M\right]\right)=$ $\left\{\left[i^{\prime}, j^{\prime}, e N_{2} ; N\right]|e|(N / M), v_{p}\left(\left(e, N_{1}\right)\right)=0\right.$ for any $p$ with $v_{p}\left(N_{2}\right)<v_{p}(M), i^{\prime} \equiv i$ $\left.\left(\bmod e N_{2}\right), j^{\prime} \equiv j\left(\bmod N_{1} / e\right)\right\}$. 
(3) Let e $\left|N, N_{2}\right|(N / e)$. Let $\rho_{e}$ be the surjective map of the modular curve of $\Gamma_{1}(N)$ onto that of $\Gamma_{1}(N / e)$ associated with $z \rightarrow e z$. Then $\rho_{e}^{-1}\left(\left[i, j, N_{2} ; N / e\right]\right)=$ $\left\{\left[i^{\prime}, j^{\prime}, e^{\prime} N_{2} ; N\right]\left|e^{\prime}\right| e,\left(e / e^{\prime}, N_{2}\right)=1, i^{\prime} e \equiv i\left(\bmod e^{\prime} N_{2}\right), j^{\prime} \equiv j\left(\bmod N / e^{\prime} N_{2}\right)\right\}$.

The lemma is easy to show, and so we skip the proof.

Now we consider Eisenstein series for $\Gamma_{1}(N)$. The result is an imitation of Hecke [2], Sect. 1 and 2. So we omit the detail. Let $k \in N$. Let $N=N_{1} N_{2}$ $\left(N_{1}, N_{2} \in \boldsymbol{N}\right)$, and $a_{1}, a_{2} \in \boldsymbol{Z}$. Then we put

$$
E_{k}\left(z, a_{1}, a_{2} / N_{2}, N_{1}\right):=\left.\sum_{\substack{m_{1} \equiv a_{1}\left(\bmod N_{1}\right) \\ m_{2} \equiv a_{2} / N_{2}(\bmod 1)}}^{\prime}\left(m_{1} z+m_{2}\right)^{-k}\left|m_{1} z+m_{2}\right|^{-s}\right|_{s=0},
$$

where the summation is over the pairs of numbers $\left(m_{1}, m_{2}\right)$ such that $m_{1}$ runs through all the integers satisfying the congruence and $m_{2}$ runs through all the rational numbers such that $m_{2}-a_{2} / N_{2} \in \boldsymbol{Z}$, and where the notation $\sum^{\prime}$ indicates as usual, that $\left(m_{1}, m_{2}\right)=(0,0)$ is to be omitted. This is an element of $E_{k}\left(\Gamma_{1}(N)\right)$ if $k \neq 2$. The space $E_{k}\left(\Gamma_{1}(N)\right)$ is zero if $k$ is odd and $N=1,2$. We exclude these cases from out argument. We have a Fourier expansion

$$
\begin{aligned}
E_{k}\left(z, a_{1}, a_{2} / N_{2}, N_{1}\right)= & \left.N^{k} \sum_{m \equiv a_{2}(\bmod N)}^{\prime} m^{-k}|m|^{-s}\right|_{s=0}\left(a_{1} \in N_{1} \boldsymbol{Z}\right) \\
& +\frac{(-2 \sqrt{-1} \pi)^{k}}{(k-1) !} \sum_{n=1}^{\infty} \sum_{\substack{n / m \equiv a_{1}\left(\bmod N_{1}\right) \\
m \in \boldsymbol{Z}}} \operatorname{sgn}(m) \boldsymbol{e}\left(a_{2} m / N_{2}\right) m^{k-1} \boldsymbol{e}(n z),
\end{aligned}
$$

where there is an additional term $-\left.\sqrt{-1} \pi \sum_{m \equiv a_{1}\left(\bmod N_{1}\right)}^{\prime} \operatorname{sgn}(m)|m|^{-s}\right|_{s=0}$ (resp. $\left.\pi /\left(N_{1} \operatorname{Im}(z)\right)\right)$ if $k=1$ (resp. $\left.k=2\right)$ and where the first term appears if the condition $a_{1} \in N_{1} Z$ is satisfied. When $k=2$, the differences of Eisenstein series are contained in $E_{2}\left(\Gamma_{1}(N)\right)$. If $e$ is a common divisor of $a_{1}, N_{1}$, then $E_{k}\left(z, a_{1}, a_{2} / N_{2}, N_{1}\right)=E_{k}\left(e z, a_{1} / e, a_{2} / N_{2}, N_{1} / e\right)$.

Suppose that $\left(a_{1}, N_{1}\right)=\left(a_{2}, N_{2}\right)=1$. Put

$$
E_{k}^{*}\left(z, a_{1}, a_{2} / N_{2}, N_{1}\right):=\left.\sum_{\substack{m_{1} \equiv a_{1}\left(\bmod N_{1}\right) \\ m_{2} \equiv a_{2} / N_{2}(\bmod 1) \\\left(m_{1}, N_{2} m_{2}\right)=1}}^{\prime}\left(m_{1} z+m_{2}\right)^{-k}\left|m_{1} z+m_{2}\right|^{-s}\right|_{s=0} .
$$

It is easily shown to be a modular form of weight $k$ for $\Gamma_{1}(N)$ if $k \neq 2$. If $k=2$, then it satisfies the same transformation law as a modular form of weight 2 , but it is not holomorphic. When $k \geq 2$, it vanishes at all the cusps of $\Gamma_{1}(N)$ but $\left[a_{2}, a_{1}, N_{2} ; N\right]$. In particular $\left\{E_{k}^{*}\left(z, a_{1}, a_{2} / N_{2}, N_{1}\right) \mid N_{1} N_{2}=N,\left(a_{1}, N_{1}\right)=\left(a_{2}, N_{2}\right)=\right.$ $1\}$ spans $E_{k}\left(\Gamma_{1}(N)\right)$ for $k \geq 3$. The Eisenstein series $E_{k}\left(z, a_{1}, a_{2} / N_{2}, N_{1}\right)$ (resp. 
$\left.E_{k}^{*}\left(z, a_{1}, a_{2} / N_{2}, N_{1}\right)\right)$ is written as a linear combination of $E_{k}^{*}\left(z, t a_{1}, t a_{2} / N_{2}, N_{1}\right)$ (resp. $\left.E_{k}\left(z, t a_{1}, t a_{2} / N_{2}, N_{1}\right)\right)$ with $t \in(Z / N)^{\times}$. Let us denote by $X_{k, N_{1}, N_{2}}$, the set $\left\{E_{k}\left(z, a_{1}, a_{2} / N_{2}, N_{1}\right) \mid\left(a_{1}, a_{2}\right) \in\left(\boldsymbol{Z} / N_{1}\right)^{\times} \times\left(\boldsymbol{Z} / N_{2}\right)^{\times} /\{ \pm 1\}\right\}$, and by $X_{k, N}$, the union $\bigcup_{N_{1} N_{2}=N} X_{k, N_{1}, N_{2}}$.

Proposition 1. Let $N, k \in N$. (1) Let $k \geq 2$. Then Eisenstein series in $X_{k, N_{1}, N_{2}}$ separate the cusps $\left[a_{2}, a_{1}, N_{2} ; N\right],\left(a_{1}, a_{2}\right) \in\left(\boldsymbol{Z} / N_{1}\right)^{\times} \times\left(\boldsymbol{Z} / N_{2}\right)^{\times} /\{ \pm 1\}$ and vanish at all other cusps. Eisenstein series in $X_{k, N}$ separate all the cusps of $\Gamma_{1}(N)$.

(2) Let $k \geq 3$. The space $E_{k}\left(\Gamma_{1}(N)\right)$ is spanned by $X_{k, N}$. The dimension is equal to 0 if $k$ is odd and $N=1,2$ and to $v_{1}(N)$ if otherwise.

(3) Let $k=2$. Then linear combinations of elements in $X_{2, N}$ which is holomorphic, span $E_{2}\left(\Gamma_{1}(N)\right)$ whose dimension is $v_{1}(N)-1$.

(4) Let $k=1$ and $N \geq 3$. Then $X_{1, N}$ spans $E_{k}\left(\Gamma_{1}(N)\right)$ whose dimension is $v_{1}(N) / 2$.

2. We introduce Eisenstein series which are suitable for study of Shimura lifting in our method. Let $N=N_{1} N_{2}$ as above. For $a_{1}, a_{2} \in Z$, let

$$
\begin{aligned}
G_{k}(z, & \left.a_{1}, a_{2}, N_{1}, N_{2}\right) \\
:= & \left.\frac{2(k-1) !}{(2 \sqrt{-1} \pi)^{k} N_{2}} \sum_{\substack{m_{1} \equiv-a_{1}\left(\bmod N_{1}\right) \\
m_{2} \in\left(1 / N_{2}\right) Z}}^{\prime} e\left(a_{2} m_{2}\right)\left(m_{1} z+m_{2}\right)^{-k}\left|m_{1} z+m_{2}\right|^{-s}\right|_{s=0} \\
= & \frac{2(k-1) !}{(2 \sqrt{-1} \pi)^{k} N_{2}} \sum_{a: Z / N_{2}} e\left(\frac{a a_{2}}{N_{2}}\right) E_{k}\left(z,-a_{1}, a / N_{2}, N_{1}\right) \\
= & \left.\sum_{m \equiv a_{2}\left(\bmod N_{2}\right)}^{\prime} \operatorname{sgn}(m)|m|^{k-1-s}\right|_{s=0} \quad\left(a_{1} \in N_{1} Z \text { and } 2 \nmid k\right) \\
& +\left.\sum_{m \equiv a_{2}\left(\bmod N_{2}\right)}^{\prime}|m|^{k-1-s}\right|_{s=0} \quad\left(a_{1} \in N_{1} \boldsymbol{Z} \text { and } 2 \mid k\right) \\
& +\frac{1}{2 \pi N_{1} y}\left(k=2 \text { and } N_{2}=1\right) \\
& +\left.\sum_{m \equiv a_{1}\left(\bmod N_{1}\right)}(\operatorname{sgn} m)|m|^{-s}\right|_{s=0} \quad\left(k=1 \text { and } N_{2}=1\right) \\
& +2 \sum_{n=1}^{\infty} \sum_{\substack{m \equiv a_{2}\left(\bmod N_{2}\right) \\
n / m \equiv a_{1}\left(\bmod N_{1}\right) \\
m \in Z}} \operatorname{sgn}(m) m^{k-1} e(n z),
\end{aligned}
$$


where the first and forth terms should be understood to be special values of partial zeta functions at non-positive integers. Obviously $G_{k}\left(z, a_{1}, a_{2}, N_{1}, N_{2}\right)=$ $G_{k}\left(z, a_{1}^{\prime}, a_{2}^{\prime}, N_{1}, N_{2}\right)$ for $a_{1} \equiv a_{1}^{\prime}\left(\bmod N_{1}\right), a_{2} \equiv a_{2}^{\prime}\left(\bmod N_{2}\right)$, and $G_{k}\left(z,-a_{1},-a_{2}\right.$, $\left.N_{1}, N_{2}\right)=(-1)^{k} G_{k}\left(z, a_{1}, a_{2}, N_{1}, N_{2}\right)$. For $M=\left(\begin{array}{ll}a & b \\ c & d\end{array}\right) \in \Gamma_{0}(N)$, there holds $\left.G_{k}\left(z, a_{1}, a_{2}, N_{1}, N_{2}\right)\right|_{M}=G_{k}\left(z, a a_{1}, d a_{2}, N_{1}, N_{2}\right)$. If $e$ is a common divisor of $a_{1}, N_{1}$ (resp. $a_{2}, N_{2}$ ), then $G_{k}\left(z, a_{1}, a_{2}, N_{1}, N_{2}\right)$ is equal to $G_{k}\left(e z, a_{1} / e, a_{2}, N_{1} / e, N_{2}\right)$ (resp. $\left.e^{k-1} G_{k}\left(e z, a_{1}, a_{2} / e, N_{1}, N_{2} / e\right)\right)$.

Let $\chi^{\prime} \in\left(\boldsymbol{Z} / N_{1}\right)^{*}, \chi \in\left(\boldsymbol{Z} / N_{2}\right)^{*}$. We define an arithmetic function $\sigma_{k, \chi}^{\chi^{\prime}}$ by setting

$$
\sigma_{k, \chi}^{\chi^{\prime}}(n)=\sum_{0<d \mid n} \chi^{\prime}(n / d) \chi(d) d^{k}, \quad n \in N
$$

Further we define $\sigma_{k, \chi}^{\chi^{\prime}}(n)$ to be 0 if $n \notin N \cup\{0\}$. If $N_{1}=1$ (resp. $N_{2}=1$ ), then we denote it by $\sigma_{k, \chi}\left(\right.$ resp. $\left.\sigma_{k}^{\chi^{\prime}}\right)$. Now assume that $\chi \chi^{\prime}$ has the same parity as $k$, namely $\chi \chi^{\prime}(-1)=(-1)^{k}$. Then we put

$$
\begin{aligned}
G_{k, \chi}^{\chi^{\prime}}(z):= & \frac{1}{2} \sum_{\substack{a_{1}:\left(\boldsymbol{Z} / N_{1}\right) \\
a_{2}:\left(\boldsymbol{Z} / N_{2}\right)}} \chi^{\prime}\left(a_{1}\right) \chi\left(a_{2}\right) G_{k}\left(z, a_{1}, a_{2}, N_{1}, N_{2}\right) \\
= & L(1-k, \chi)\left(N_{1}=1\right)+\frac{1}{4 \pi N_{1} y} \sum_{a_{1}:\left(\boldsymbol{Z} / N_{1}\right)} \chi^{\prime}\left(a_{1}\right) \quad\left(k=2 \text { and } N_{2}=1\right) \\
& +(\sqrt{-1} \pi)^{-1} L\left(1, \chi^{\prime}\right) \quad\left(k=1 \text { and } N_{2}=1\right)+2 \sum_{n=1}^{\infty} \sigma_{k-1, \chi}^{\chi^{\prime}}(n) \boldsymbol{e}(n z) .
\end{aligned}
$$

For $k=1, G_{1, \chi}^{\chi^{\prime}}(z)=G_{1, \chi^{\prime}}^{\chi}(z)$. If $N_{1}=1$ (resp. $N_{2}=1$ ), then we denote the Eisenstein series by $G_{k, \chi}(z)$ (resp. $G_{k}^{\chi^{\prime}}(z)$ ). If $N_{1}=N_{2}=1$, then it is denoted by $G_{k}(z)$. We define $\sigma_{k-1, \chi}^{\chi^{\prime}}(0)$ to be the half of the constant term of the Fourier expansion of $G_{k, \chi}^{\chi^{\prime}}(z)$ at the cusp $\sqrt{-1} \infty$. Hence it is 0 if $N_{1}>0$ and $k>1$. Let us set

$$
Y_{k, N_{1}, N_{2}}:=\left\{G_{k, \chi}^{\chi^{\prime}}(e z)|e| N_{2}, \chi^{\prime} \in\left(Z / N_{1}\right)^{*}, x \in\left(Z /\left(N_{2} / e\right)\right)^{*}, \chi \chi^{\prime}(-1)=(-1)^{k}\right\} .
$$

The Eisenstein series $G_{k, \chi}^{\chi^{\prime}}(e z)$ in $Y_{k, N_{1}, N_{2}}$ is holomorphic if and only if $k \neq 2$ or it is not in the form $G_{2}^{\chi^{\prime}}\left(N_{2} z\right)$ with $\chi^{\prime}=1_{N_{1}}$.

Lemma 2. Let $k \geq 2$. Let $N=N_{1} N_{2}$. Then the $C$-span of $Y_{k, N_{1}, N_{2}}$ is equal to the C-span of $X_{k, N_{1}, M_{2}}$ 's, $M_{2} \mid N_{2}$. 
Proof. By definition, the former is obviously contained in the latter. We must show the converse. Let $a_{1} \in\left(Z / N_{1}\right), a \in Z$. Then

$$
\begin{aligned}
G_{k}\left(z, a_{1}, a, N_{1}, N_{2}\right) & \\
= & \left(a, N_{2}\right)^{k-1} G_{k}\left(\left(a, N_{2}\right) z, a_{1}, a /\left(a, N_{2}\right), N_{1}, N_{2} /\left(a, N_{2}\right)\right) \\
= & 2\left(a, N_{2}\right)^{k-1} \varphi\left(N_{1}\right)^{-1} \varphi\left(N_{2}\right)^{-1} \\
& \times \sum_{\substack{\chi^{\prime} \in\left(\boldsymbol{Z} / N_{1}\right)^{*} \\
\chi \in\left(\boldsymbol{Z} /\left(N_{2} /\left(a, N_{2}\right)\right)^{*}\right.}} \chi^{\prime}\left(a_{1}\right)^{-1} \chi\left(a /\left(a, N_{2}\right)\right)^{-1} G_{k, \chi}^{\chi^{\prime}}\left(\left(a, N_{2}\right) z\right) .
\end{aligned}
$$

Hence the $C$-span of $Y_{k, N_{1}, N_{2}}$ contains $G_{k}\left(z, a_{1}, a, N_{1}, N_{2}\right), a_{1} \in\left(Z, N_{1}\right), a \in Z$. For $a_{2} \in Z$ we have

$$
E_{k}\left(z,-a_{1}, a_{2} / N_{2}, N_{1}\right)=\frac{(2 \sqrt{-1} \pi)^{k}}{2(k-1) !} \sum_{a \in Z / N_{2}} e\left(-\frac{a a_{2}}{N_{2}}\right) G_{k}\left(z, a_{1}, a, N_{1}, N_{2}\right)
$$

This shows our assertion.

q.e.d.

Corollary. Let $k \geq 2$. Let $N=N_{1} N_{2}$.

(1) An Eisenstein series in $Y_{k, N_{1}, N_{2}}$ vanishes at cusps $\left[*, *, N_{2}^{\prime} ; N\right]$ for $N_{2}^{\prime}$ with $N_{2}^{\prime} \Varangle N_{2}$.

(2) The elements of $Y_{k, N_{1}, N_{2}}$ separate cusps $\left[a_{1}, a_{2}, N_{2} ; N\right]\left(a_{1} \in\left(Z / N_{1}\right), a_{2} \in\right.$ $\left.\left(Z / N_{2}\right)^{\times}\right)$of $\Gamma_{1}(N)$.

Proof. Let $M_{2}$ be a divisor of $N_{2}$. An Eisenstein series in $X_{k, N_{1}, M_{2}}$ vanishes at $\left[*, *, N_{2}^{\prime} ; N_{1} M_{2}\right]$ with $N_{2}^{\prime} \nmid M_{2}$ by Proposition 1 (2). Hence by Lemma 1 (2), it does at $\left[*, *, N_{2}^{\prime} ; N\right]$ with $N_{2}^{\prime} \Varangle N_{2}$. This shows the first assertion. The second assertion follows from Proposition 1 (1).

q.e.d.

Lemma 3. Let $G_{k, \chi}^{\chi^{\prime}}(e z) \in Y_{k, N_{1}, N_{2}}$. Suppose that $\chi$ is not a primitive character $\left(\bmod N_{2} / e\right)$. Then one of the following holds;

(1) $G_{k, \chi}^{\chi^{\prime}}(e z)$ vanishes at cusps $\left[*, *, N_{2} ; N\right]$, or

(2) there is $G_{k, \omega}^{\chi^{\prime}}\left(e e^{\prime} z\right) \in Y_{k, N_{1}, N_{2}}$ with $e^{\prime}>1, \omega \in\left(Z /\left(N_{2} / e e^{\prime}\right)\right)^{*}$ whose constant multiple takes the same value as $G_{k, \chi}^{\chi^{\prime}}(e z)$ at each cusp $\left[*, *, N_{2} ; N\right]$.

Proof. Let $M_{2}$ be a conductor of $\chi$, which is a proper divisor of $N_{2} / e$, and let $\omega$ be the primitive character $\left(\bmod M_{2}\right)$ associated with $\chi$. There are two cases that (i) $v_{p}\left(M_{2}\right)>0$ for any prime factor $p$ of $N_{2} / e$, and that (ii) there is a prime 
factor $p$ of $N_{2} / e$ with $v_{p}\left(M_{2}\right)=0$. Let us consider the case (i). Then $G_{k, \chi}^{\chi^{\prime}}(e z)=$ $G_{k, \omega}^{\chi^{\prime}}(e z)$, and it is in $Y_{k, N_{1}, e M_{2}}$, or in the linear span of $X_{k, N_{1}, c M_{2}}$. Then by Proposition 1 (1), it vanishes at cusps $\left[*, *, N_{2}^{\prime} ; N\right]$ with $N_{2}^{\prime} \Varangle e M_{2}$, in particular at $\left[*, *, N_{2} ; N\right]$. Now we consider the case (ii). Let $\left\{p_{1}, \ldots, p_{s}\right\}$ be all the prime factors of $N_{2} / e$ relatively prime to $M_{2}$. Then we have equalities

$$
\begin{aligned}
\sigma_{k, \omega}^{\chi^{\prime}}(n)= & \sigma_{k, \chi}^{\chi^{\prime}}(n)+\sum_{i=1}^{s} p_{i}^{k-1} \omega\left(p_{i}\right) \sigma_{k, \omega}^{\chi^{\prime}}\left(n / p_{i}\right) \\
& -\sum_{1 \leq i, j \leq s}\left(p_{i} p_{j}\right)^{k-1} \omega\left(p_{i} p_{j}\right) \sigma_{k, \omega}^{\chi^{\prime}}\left(n / p_{i} p_{j}\right) \\
& +\cdots+(-1)^{s}\left(p_{1} \cdots p_{s}\right)^{k-1} \omega\left(p_{1} \cdots p_{s}\right) \sigma_{k, \omega}^{\chi^{\prime}}\left(n / p_{1} \cdots p_{s}\right)
\end{aligned}
$$

for $n \in N$ and

$$
\begin{aligned}
G_{k, \chi}^{\chi^{\prime}}(e z)= & G_{k, \omega}^{\chi^{\prime}}(e z)-\sum_{i=1}^{s} p_{i}^{k-1} \omega\left(p_{i}\right) G_{k, \omega}^{\chi^{\prime}}\left(p_{i} e z\right) \\
& +\sum_{1 \leq i, j \leq s}\left(p_{i} p_{j}\right)^{k-1} \omega\left(p_{i} p_{j}\right) G_{k, \omega}^{\chi^{\prime}}\left(p_{i} p_{j} e z\right) \\
& -\cdots+(-1)^{s+1}\left(p_{1} \cdots p_{s}\right)^{k-1} \omega\left(p_{1} \cdots p_{s}\right) G_{k, \omega}^{\chi^{\prime}}\left(p_{1} \cdots p_{s} e z\right)
\end{aligned}
$$

Except for the last one, Eisenstein series of the right hand side vanish at cusps $\left[*, *, N_{2} ; N\right]$ since they are in $Y_{k, N_{1}, N_{2}^{\prime \prime}}$ with proper divisors $N_{2}^{\prime \prime}$ of $N_{2}$. If the last one vanishes, the $G_{k, \chi}^{\chi^{\prime}}(e z)$ does also. The last one does not vanish only when $N_{2}=p_{1} \cdots p_{s} e M_{2}$. Since $G_{k, \chi}^{\chi^{\prime}}(e z)$ is equal to $G_{k, \omega}^{\chi^{\prime}}\left(p_{1} \cdots p_{s} e z\right) \in Y_{k, N_{1}, N}$ up to a constant multiple, our assertion is proved.

q.e.d.

Let us set

$$
\begin{aligned}
Z_{k, N_{1}, N_{2}}:= & \left\{G_{k, \chi}^{\chi^{\prime}}(e z)|e| N_{2}, \chi^{\prime} \in\left(\boldsymbol{Z} / N_{1}\right)^{*},\right. \\
& \text { primitive } \left.\chi \in\left(\boldsymbol{Z} /\left(N_{2} / e\right)\right)^{*},\left(\chi^{\prime} \chi\right)(-1)=(-1)^{k}\right\} .
\end{aligned}
$$

The set $Z_{k, N_{1}, N_{2}}$ is a subset of $Y_{k, N_{1}, N_{2}}$. However as Lemma 3 shows, the separations of cusps $\left[*, *, N_{2} ; N\right]$ by elements in $Z_{k, N_{1}, N_{2}}$, and by elements in $Y_{k, N_{1}, N_{2}}$, are the same.

Here we note that if $v_{2}\left(N_{2} / e\right)=1$, then no characters in $\left(Z /\left(N_{2} / e\right)\right)^{*}$ are primitive. 
Proposition 2. Let $k \geq 2$ and let $k_{0} \geq 1$. Let $S$ be a set of some divisors of $N$. Let $f_{e, N_{2}}\left(N_{2} \in S, e \mid N_{2}\right)$ be modular forms for $\Gamma_{1}(N)$ of weight $k_{0}$ such that $f_{e, N_{2}}$ takes nonzero values at cusps $\left[*, *, N_{2} ; N\right]$. Then $\left\{f_{e, N_{2}}(z) G_{k, \chi}^{\chi^{\prime}}(e z) \mid N_{1} N_{2}=N\right.$, $\left.N_{2} \in S, G_{k, \chi}^{\chi^{\prime}}(e z) \in Z_{k, N_{1}, N_{2}}\right\}$ separates the cusps $\left[*, *, N_{2} ; N\right]\left(N_{2} \in S\right)$. If $S$ is the set of all divisors of $N$, then it separates all the cusps of $\Gamma_{1}(N)$.

Proof. Let $r, s$ be two distinct cusps of $\Gamma_{1}(N)$. At first suppose that they are in the form $r=\left[*, *, N_{2} ; N\right], s=\left[*, *, N_{2}^{\prime} ; N\right]$ with $N_{2} \neq N_{2}^{\prime}, N_{2}, N_{2}^{\prime} \in S$. Replacing $N_{2}$ and $N_{2}^{\prime}$ if necessary, we may assume that $N_{2}^{\prime} \nmid N_{2}$. Then Corollary to Lemma 2 shows that there is an element of $Y_{k, N_{1}, N_{2}}$ which vanishes at $s$ and does not vanish at $r$. By Lemma 3 such an element exists also in $Z_{k, N_{1}, N_{2}}$. Then the assertion immediately follows in this case. Now suppose that $r, s$ are both in the form $\left[*, *, N_{2} ; N\right]$. Let $e$ be a maximal divisor of $N_{2}$ so that $\rho_{e}(r) \neq \rho_{e}(s)$ (see Lemma 1 (3)). By Proposition 1 (1), $E_{k}\left(z,-a_{1}, a_{2} /\left(N_{2} / e\right), N_{1}\right), a_{1} \in\left(Z / N_{1}\right), a_{2} \in$ $\left(\boldsymbol{Z} /\left(N_{2} / e\right)\right)$, separate $\rho_{e}(r)$ and $\rho_{e}(r)$. They are written as linear combinations of $G_{k, \chi}^{\chi^{\prime}}\left(e^{\prime \prime} z\right), \chi^{\prime} \in\left(\boldsymbol{Z} / N_{1}\right)^{*}, e^{\prime \prime} \mid\left(N_{2} / e\right), \chi \in\left(\boldsymbol{Z} /\left(N_{2} / e e^{\prime \prime}\right)\right)^{*}$. The maximality of $e$ implies that each $G_{k, \chi}^{\chi^{\prime}}\left(e^{\prime \prime} z\right)\left(e^{\prime \prime}>1\right)$ takes the same value at $\rho_{e}(r)$ and at $\rho_{e}(s)$. By Lemma $3 G_{k, \chi}^{\chi^{\prime}}(z)$ with $\chi \in\left(Z /\left(N_{2} / e\right)\right)^{*}$ not primitive, also takes the same value. It follows that $G_{k, \chi}^{\chi^{\prime}}(z)$ 's, with primitive $\chi \in\left(Z /\left(N_{2} / e\right)\right)^{*}$, separate these two cusps. Hence $G_{k, \chi}^{\chi^{\prime}}(e z)^{\prime}$ 's, with $\chi^{\prime} \in\left(\boldsymbol{Z} / N_{1}\right)^{*}$ and primitive $\chi \in\left(\boldsymbol{Z} /\left(N_{2} / e\right)\right)^{*}$, separate $r$ and $s$. Since $f_{e, N_{2}}(z)$ vanishes at neither $r$ nor $s, f_{e, N_{2}}(z) G_{k, \chi}^{\chi^{\prime}}(e z)$ 's separate $r$ and $s$. This shows our assertion.

q.e.d.

Take characters $\chi^{\prime} \in\left(\boldsymbol{Z} / N_{1}\right)^{*}$ and $\chi \in\left(\boldsymbol{Z} /\left(N_{2} / e\right)\right)^{*}$. Let $\chi_{0}:=\chi^{\prime} \chi$ be a character $(\bmod N)$. Then obviously $G_{k, \chi}^{\chi^{\prime}}(e z)$ satisfies

$$
\left.G_{k, \chi}^{\chi^{\prime}}(e z)\right|_{M, \chi_{0}}=G_{k, \chi}^{\chi^{\prime}}(e M z), \quad M \in \Gamma_{0}(N),
$$

namely it is in $M_{k}\left(N, \chi_{0}\right)$. As a corollary to the above proof, we obtain the following;

COROLlary 1. (1) Suppose that $k \neq 2$ or $\chi_{0} \neq 1_{N}$. Then $\left\{G_{k, \chi}^{\chi^{\prime}}(e z) \mid N_{1} N_{2}=N\right.$, $e \mid N_{2}, \chi^{\prime} \in\left(\boldsymbol{Z} / N_{1}\right)^{*}$, primitive $\left.\chi \in\left(\boldsymbol{Z} /\left(N_{2} / e\right)\right)^{*},\left(\chi^{\prime} \chi\right)^{(e)}=\chi_{0}\right\}$ forms a basis of $E_{k}\left(N, \chi_{0}\right)$.

(2) Let $k=2$. Then $\left\{G_{2, \chi}^{\chi^{\prime}}(e z)\left|N_{1} N_{2}=N, e\right| N_{2}, e \neq N_{2}, \chi^{\prime} \in\left(Z / N_{1}\right)^{*}\right.$, primitive $\left.\chi \in\left(Z /\left(N_{2} / e\right)\right)^{*},\left(\chi^{\prime} \chi\right)^{(e)}=1_{N}\right\} \cup\left\{G_{2}\left(N_{2} z\right)-(1 / N)_{2} G_{2}(z)\left|N_{2}\right| N\right\}$ is a basis of $E_{2}\left(N, 1_{N}\right)$. 
ProOF. In the proof of Lemma 2 we showed that $E_{k}\left(z,-a_{1}, a_{2} / N_{2}, N\right)$ is written as a linear combination of $G_{k, \chi}^{\chi^{\prime}}(e z)$ in $\bigcup_{N_{1} N_{2}=N} Y_{k, N_{1}, N_{2}}$. In the proof of Lemma 3, we actually showed that $G_{k, \chi}^{\chi^{\prime}}(e z)$ with $\chi$ not primitive, is written as a linear combinations of ones with primitive $\chi$. The argument is valid also for $k=1$. By Proposition 1, $\bigcup_{N_{1} N_{2}=N} Z_{k, N_{1}, N_{2}}$ spans $E_{k}\left(\Gamma_{1}(N)\right)$ for $k \neq 2$. For $k=2$, linear combinations of elements in $Z_{2, N_{2}, N_{2}}$ 's which are holomorphic, span $E_{2}\left(\Gamma_{1}(N)\right)$. In the former case $\bigcup_{N_{1} N_{2}=N} Z_{k, N_{1}, N_{2}}$ forms a basis since the number of elements equals the dimension, and in the latter, $\bigcup_{N_{1} N_{2}=N}\left[Z_{2, N_{1}, N_{2}}-\left\{G_{2}^{1_{N_{1}}}\left(N_{2} z\right)\right\}\right] \cup$ $\left\{G_{2}\left(N_{2} z\right)-\left(1 / N_{2}\right) G_{2}(z)\left|N_{2}\right| N\right\}$ forms a basis. The space $E_{k}\left(N, \chi_{0}\right)$ is the invariant subspace of $E_{k}\left(\Gamma_{1}(N)\right)$ under the action of $\left.f \rightarrow f\right|_{M, \chi_{0}}, M \in \Gamma_{0}(N)$, and there is the decomposition $E_{k}\left(\Gamma_{1}(N)\right)=\bigoplus_{\chi_{0}} E_{k}\left(N, \chi_{0}\right)$. If $k \neq 2$, then each element of $\bigcup_{N_{1} N_{2}=N} Z_{k, N_{1}, N_{2}}$ belongs to some $E_{k}\left(N, \chi_{0}\right)$. This shows our assertion. The case $k=2$ is similar.

q.e.d.

3. In what follows, we always assume that $N$ is divisible by 4 . Let

$$
\theta(z):=\sum_{n \in Z} \boldsymbol{e}\left(n^{2} z\right)
$$

be a theta series, which is a modular form for $\Gamma_{0}(4)$ of weight $1 / 2$. We denote by $j(M, z)\left(M \in \Gamma_{0}(4)\right)$, the automorphy factor of the theta series. Its forth power $j(M, z)^{4}$ is equal to $(c z+d)^{2}, M=\left(\begin{array}{ll}a & b \\ c & d\end{array}\right)$. For $k \in N, M_{k+1 / 2}\left(\Gamma_{1}(N)\right)$ denotes the space of modular forms for $\Gamma_{1}(N)$ with an automorphy factor $j(\boldsymbol{M}, z)$. $(c z+d)^{k}$, and $S_{k+1 / 2}\left(\Gamma_{1}(N)\right)$ denotes the subspace consisting of cusp forms. Let $\chi_{0}$ be a character $(\bmod N)$. Then $M_{k+1 / 2}\left(N, \chi_{0}\right)\left(\operatorname{resp} . S_{k+1 / 2}\left(N, \chi_{0}\right)\right)$ denotes the space of modular forms (resp. cusp forms) $f$ such that $\left(\left.f\right|_{M, \chi_{0}}\right)(z)=f(z)$ for $M \in \Gamma_{0}(N)$ where $\left(\left.f\right|_{M, \chi_{0}}\right)=\chi_{0}(d)^{-1} j(M, z)^{-1}(c z+d)^{-k} f(M z)$. It should be noted that our automorphy factor differs from Shimura's in [7] by $\chi_{-4}(d)^{k}$ where $\chi_{-4}$ is the Kronecker-Jacobi-Legendre symbol. Let $e \in N$. Then $\theta(e z)$ is in $M_{1 / 2}\left(4 e,\left(\frac{e}{\mid l}\right)\right)$, indeed we have

$$
j\left(\left(\begin{array}{cc}
a & e b \\
c / e & d
\end{array}\right), e z\right)=\left(\frac{e}{|d|}\right) j\left(\left(\begin{array}{ll}
a & b \\
c & d
\end{array}\right), z\right), \quad\left(\begin{array}{ll}
a & b \\
c & d
\end{array}\right) \in \Gamma_{0}(4 e) .
$$

The group $\Gamma_{0}(4)$ has three cusps $0,1 / 2,1 / 4$, and $\theta(z)$ vanishes only at the 
cusp $1 / 2$. Then $\theta(e z)$ vanishes only at the cusps $i / 2 e,(i, 2 e)=1$, of $\Gamma_{0}(4 e)$, or equivalently only at cusps $[*, *, 2 e ; 4 e]$ of $\Gamma_{1}(4 e)$.

Let $v_{2}(N)=2$. Then cusps in the form $\left[*, *, N_{2} ; N\right]$ with $v_{2}\left(N_{2}\right)=1$ is irregular for $\Gamma_{1}(N)$ and for an automorphy factor $j(M, z)(c z+d)^{k}, \quad M=$ $\left(\begin{array}{ll}a & b \\ c & d\end{array}\right) \in \Gamma_{1}(N)$ (cf. Shimura [6]), and so any modular forms for $\Gamma_{1}(N)$ with the automorphy factor vanishes at those cusps. Then other cusps are all regular. If $v_{2}(N)>2$, then all cusps are regular.

Proposition 3. Let $4 \mid N$. Let $\phi_{4}$ denote an arithmetic function defined by $\phi_{4}(e)=e / 4\left(v_{2}(e) \geq 2\right), e\left(v_{2}(e) \leq 1\right)$.

(1) Let $k \geq 2$. If $v_{2}(N)=2$, then $\left\{\theta\left(\phi_{4}(e) z\right) G_{k, \chi}^{\chi^{\prime}}(e z) \mid N_{1} N_{2}=N, v_{2}\left(N_{2}\right) \neq 1\right.$, $\left.G_{k, \chi}^{\chi^{\prime}}(e z) \in Z_{k, N_{1}, N_{2}}\right\}$ separates all the regular cusps of $\Gamma_{1}(N)$. If $v_{2}(N)>2$, then $\left\{\theta\left(\phi_{4}(e) z\right) G_{k, \chi}^{\chi^{\prime}}(e z) \mid N_{1} N_{2}=N, G_{k, \chi}^{\chi^{\prime}}(e z) \in Z_{k, N_{1}, N_{2}}\right\}$ separates all the cusps of $\Gamma_{1}(N)$.

(2) Let $k=2$. If $v_{2}(N)=2$, then $\left\{\theta\left(\phi_{4}(e) z\right) G_{2, \chi}^{\chi^{\prime}}(e z) \mid N_{1} N_{2}=N, v_{2}\left(N_{2}\right) \neq 1\right.$, $G_{2, \chi}^{\chi^{\prime}}(e z) \in Z_{2, N_{1}, N_{2}}, e<N_{2}$ or $\left.\left.\chi^{\prime} \neq 1_{N_{1}}\right\} \cup\left\{\theta\left(\phi_{4}\left(N_{2}\right) z\right)\left(G_{2}^{1_{N_{1}}}\left(N_{2} z\right)-G_{2}^{1_{2 N_{1}}}\left(N_{2} / 2\right) z\right)\right)\right\}$ $\left.N_{1} N_{2}=N, 4 \mid N_{2}\right\} \cup\left\{\theta\left(N_{2} z\right)\left(2 G_{2}^{1_{\left(N_{1} / 2\right)}}\left(2 N_{2} z\right)-G_{2}^{1_{N_{1}}}\left(N_{2} z\right)\right) \mid N_{1} N_{2}=N, 2 \nmid N_{2}\right\}$ separates the regular cusps of $\Gamma_{1}(N)$. If $v_{2}(N)>2$, then $\left\{\theta\left(\phi_{4}(e) z\right) G_{2, \chi}^{\chi^{\prime}}(e z) \mid\right.$ $N_{1} N_{2}=N, G_{2, \chi}^{\chi^{\prime}}(e z) \in Z_{2, N_{1}, N_{2}}, e<N_{2}$ or $\left.\chi^{\prime} \neq 1_{N_{1}}\right\} \cup\left\{\left(\theta\left(\phi_{4}\left(N_{2}\right) z\right)\left(2 G_{2}^{1_{N_{1}}}\left(N_{2} z\right)-\right.\right.\right.$ $\left.\left.G_{2}^{1_{2 N_{1}}}\left(\left(N_{2} / 2\right) z\right)\right)\left|N_{1} N_{2}=N, 4\right| N_{2}\right\} \cup\left\{\theta\left(N_{2} z\right)\left(2 G_{2}^{1_{\left(N_{1} / 2\right)}}\left(2 N_{2} z\right)-G_{2}^{1_{N_{1}}}\left(N_{2} z\right)\right) \mid N_{1} N_{2}=\right.$ $\left.N, v_{2}\left(N_{2}\right) \leq 1\right\}$ separates all the cusps of $\Gamma_{1}(N)$. All the elements in the sets are holomorphic.

Proof. (1) If $G_{k, \chi}^{\chi^{\prime}}(e z) \in Z_{k, N_{1}, N_{2}}$, then $v_{2}\left(N_{2} / e\right) \neq 1$. So theta series $\theta\left(\phi_{4}(e) z\right)$ does not vanish at cusps $i / N_{2}\left(\left(i, N_{2}\right)=1\right)$. Then we can apply Proposition 2 to our case. Then the assertion follows.

(2) Let $v_{2}\left(N_{2}\right)>0$. Let $b$ be 1 or 2 according as $N_{1}$ is odd or even. Then $G_{2}^{1_{N_{1}}}\left(N_{2} z\right)$ and $\left.1 / 2\left\{b G_{2}^{1_{N_{1}}}\left(N_{2} z\right)-G_{2}^{1_{2 N_{1}}}\left(\left(N_{2} / 2\right) z\right)\right)\right\}$ takes the same value at cusps $\left[*, *, N_{2} ; N\right]$ because $\left.G_{2}^{1_{2 N_{1}}}\left(\left(N_{2} / 2\right) z\right)\right)$ vanishes there. The latter vanishes at cusps $\left[*, *, N_{2}^{\prime} ; N\right]$ with $N_{2} \nmid N_{2}^{\prime}$ as well as the former. This shows that we can replace $\theta\left(\phi_{4}\left(N_{2}\right) z\right) G_{2}^{1_{N_{1}}}\left(N_{2} z\right)$ by $\left.\theta\left(\phi_{4}\left(N_{2}\right) z\right)\left(b G_{2}^{1_{N_{1}}}\left(N_{2} z\right)-G_{2}^{1_{2 N_{1}}}\left(\left(N_{2} / 2\right) z\right)\right)\right)$ in the argument $(1)$. Let $v_{2}\left(N_{2}\right) \leq 1$. Then the values at cusps $\left[*, *, N_{2} ; N\right]$ of $G_{2}^{1_{N_{1}}}\left(N_{2} z\right)$ and of $2 G_{2}^{1_{\left(N_{1} / 2\right)}}\left(2 N_{2} z\right)-G_{2}^{1_{N_{1}}}\left(N_{2} z\right)$ are proportional to each other, indeed they are modular forms for $\Gamma_{0}(N)$ with trivial character and each of them is a non-zero constant on those cusps (see Lemma 1 (1)). The modular form $G_{2}^{1_{\left(N_{1} / 2\right)}}\left(2 N_{2} z\right)$ does not vanish at cusps $\left[*, *, 2 N_{2} ; N\right]$. However a theta series $\theta\left(N_{2} z\right)$ vanishes at cusps 
$\left[*, *, 2 N_{2} ; N\right]$ and so $\theta\left(N_{2} z\right)\left(2 G_{2}^{1_{\left(N_{1} / 2\right)}}\left(2 N_{2} z\right)-G_{2}^{1_{N_{1}}}\left(N_{2} z\right)\right)$ vanishes at cusps $\left[*, *, N_{2}^{\prime} ; N\right]$ with $N_{2}^{\prime} \nmid N_{2}$. Since the values at cusps $\left[*, *, N_{2} ; N\right]$, of $\theta\left(N_{2} z\right)$. $\left.G_{2}^{1_{N_{1}}}\left(N_{2} z\right)\right)$ and of $\theta\left(N_{2} z\right)\left(2 G_{2}^{1_{\left(N_{1} / 2\right)}}\left(2 N_{2} z\right)-G_{2}^{1_{N_{1}}}\left(N_{2} z\right)\right)$ are proportional, we can replace the former by the latter in the argument (1).

q.e.d.

Let $G_{k+1 / 2}\left(\Gamma_{1}(N)\right)$ denote the subspace of $M_{k+1 / 2}\left(\Gamma_{1}(N)\right)$ generated by modular forms in Proposition 3 (1) if $k=1$ or $k>2$, and by those in Proposition 3 (2) if $k=2$. Proposition 3 implies that

$$
M_{k+1 / 2}\left(\Gamma_{1}(N)\right)=G_{k+1 / 2}\left(\Gamma_{1}(N)\right) \oplus S_{k+1 / 2}\left(\Gamma_{1}(N)\right)
$$

for $k \geq 2$. For $k \neq 2$, let $G_{k+1 / 2}\left(N, \chi_{0}\right)$ denote the linear span of $\left\{\theta\left(\phi_{4}(e) z\right) G_{k, \chi}^{\chi^{\prime}}(e z)\left|N_{1} N_{2}=N, e\right| N_{2}, \chi^{\prime} \in\left(Z / N_{1}\right)^{*}, \quad\right.$ primitive $\quad \chi \in\left(Z /\left(N_{2} / e\right)\right)^{*}$ $\chi_{0}=\left(\frac{\phi_{4}(e)}{}\right) \chi^{\prime} \chi$ on $\left.(Z / N)^{\times}\right\}$where the condition $v_{2}\left(N_{2}\right) \neq 1$ should be added if $v_{2}(N)=2$. It is a subspace of $M_{k+1 / 2}\left(N, \chi_{0}\right)$. Since the direct sum of $G_{k+1 / 2}\left(N, \chi_{0}\right)$ 's with $\chi_{0} \in(Z / N)^{*}$ is equal to $G_{k+1 / 2}\left(\Gamma_{1}(N)\right)$, we have $M_{k+1 / 2}\left(N, \chi_{0}\right)=G_{k+1 / 2}\left(N, \chi_{0}\right) \oplus S_{k+1 / 2}\left(N, \chi_{0}\right)$ for $k>2$. By the similar way we construct $G_{k+1 / 2}\left(N, \chi_{0}\right)$ also for $k=2$ by replacing $\theta\left(\phi_{4}\left(N_{2}\right) z\right) G_{2}^{1_{N_{1}}}\left(N_{2} z\right)$ by $\theta\left(\phi_{4}\left(N_{2}\right) z\right)\left\{2 G_{2}^{1_{N_{1}}}\left(N_{2} z\right)-G_{2}^{1_{2 N_{1}}}\left(\left(N_{2} / 2\right) z\right)\right\} \quad\left(v_{2}\left(N_{2}\right) \geq 2\right)$, and $\left.\theta\left(N_{2} z\right) G_{2}^{1_{N_{1}}}\left(N_{2} z\right)\right)$ by $\theta\left(N_{2} z\right)\left\{2 G_{2}^{I_{\left(N_{1} / 2\right)}}\left(2 N_{2} z\right)-G_{2}^{\Gamma_{N_{1}}}\left(N_{2} z\right)\right\} \quad\left(v_{2}\left(N_{2}\right) \leq 1\right)$ as in the proof of Proposition 3 . Then the above equality holds also for $k=2$. We obtain;

COROllary. Let $k \geq 2$. Then we have

$$
M_{k+1 / 2}\left(N, \chi_{0}\right)=G_{k+1 / 2}\left(N, \chi_{0}\right) \oplus S_{k+1 / 2}\left(N, \chi_{0}\right)
$$

4. Let $4 \mid N, \chi_{0} \in(Z / N)^{*}$. Let $p$ be prime. A Hecke operator $T_{\chi_{0}}(p)$ defines a $\boldsymbol{C}$-linear endomorphism on $M_{k}\left(N, \chi_{0}\right)$ such that for $f(z)=\sum_{n=0}^{\infty} c_{n} \boldsymbol{e}(n z)$, $\left(T_{\chi_{0}}(p) f\right)(z)=\sum_{n=0}^{\infty}\left(c_{p n}+\chi_{0}(p) p^{k-1} c_{p / n}\right) e(n z)$ where $c_{p / n}=0$ if $p / n$ is not integral. If $p \mid N$, then it is denoted also by $U_{p}$. If $v_{p}(N) \geq 2$, then $U_{p}(f)$ is in $M_{k}\left(N / p, \chi_{0}\right)$. A Hecke operator $T_{\chi_{0}}\left(p^{2}\right)$ on $M_{k+1 / 2}\left(N, \chi_{0}\right)$ is such that for $f(z)=$ $\sum_{n=0}^{\infty} c_{n} \boldsymbol{e}(n z), \quad\left(T_{\chi_{0}}\left(p^{2}\right) f\right)(z)=\sum_{n=0}^{\infty}\left(c_{p^{2} n}+\chi_{0}(p)(n / p) p^{k-1} c_{n}+\chi_{0}\left(p^{2}\right) p^{2 k-1} c_{n / p^{2}}\right)$ $\cdot e(n z)$ (Shimura [7, Prop. 1.7]). For $p \mid N$, the Hecke operator is denoted also by $U_{p^{2}}$. For $e \in N, B_{e}$ is defined to be an operator of the space of Fourier series sending $f(z)$ to $f(e z)$. We have inclusions $B_{e}\left(M_{k}\left(N, \chi_{0}\right)\right) \subset M_{k}\left(e N, \chi_{0}^{(e)}\right)$ and 
$B_{e}\left(M_{k+1 / 2}\left(N, \chi_{0}\right)\right) \subset M_{k+1 / 2}\left(e N,(e /|\cdot|) \chi_{0}\right)$ where $\chi_{0}^{(c)}$ is as in the beginning of the section one.

Let $a$ be a squarefree natural number. Let $a^{*}$ denotes $a$ or $4 a$ according as $a \equiv 1(\bmod 4)$ or not. We note that $a^{*}$ is a discriminant of a real quadratic field except for the case $a=a^{*}=1$. Let $\chi_{a^{*}}$ be as in the beginning of the section one. Let $f(z)=\sum_{n=0}^{\infty} c_{n} e(n z) \in M_{k+1 / 2}\left(N, \chi_{0}\right)$. Then we define a Shimura lifting $\mathscr{S}_{a, \chi_{0}}(f)$ of $f$ to be

$$
\mathscr{S}_{a, \chi_{0}}(f)(z)=C+\sum_{n=1}^{\infty}\left(\sum_{0<d \mid n} \chi_{a^{*}}(d) \chi_{0}(d) d^{k-1} c_{a n^{2} / d^{2}}\right) \boldsymbol{e}(n z)
$$

provided that there is a constant $C$ so that $\mathscr{S}_{a, \chi_{0}}(f)$ is a modular form for some congruence subgroup. The constant $C$ is obviously unique if it exists. Shimura [7] and Niwa [4] showed that $\mathscr{S}_{a, \chi_{0}}$ is a well-defined linear map of $S_{k+1 / 2}\left(N, \chi_{0}\right)$ to $M_{2 k}\left(N / 2, \chi_{0}^{2}\right)$, and that $\mathscr{S}_{a, \chi_{0}}(f)$ is a cusp form in $S_{2 k}\left(N / 2, \chi_{0}^{2}\right)$ for $f \in$ $S_{k+1 / 2}\left(N, \chi_{0}\right)$ and for $k \geq 2$, in particular $C=0$.

The lifting maps allow us to make formal computations for higher terms of the Fourier expansions of modular forms. In principle, higher terms determine the modular form, namely the constant term. In this sense the formal computation of the lifting map is possible as well as the Hecke operators and the operator $B_{e}$. By simple computations, we obtain the following;

LeMma 4. The Shimura lifting map $\mathscr{S}_{a, \chi_{0}}$ is Hecke equivariant map, namely there holds

$$
\begin{aligned}
& \mathscr{S}_{a, \chi_{0}} \circ T_{\chi_{0}}\left(p^{2}\right)=T_{\chi_{0}^{2}}(p) \circ \mathscr{S}_{a, \chi_{0}}, \\
& \mathscr{S}_{a, \chi_{0}} \circ U_{p^{2}}=U_{p} \circ \mathscr{S}_{a, \chi_{0}}(p \mid N) .
\end{aligned}
$$

In particular if $f \in M_{k+1 / 2}\left(N, \chi_{0}\right)$ is a common eigen-function of $T_{\chi_{0}}\left(p^{2}\right)$ for all $p$, then $\mathscr{S}_{a, \chi_{0}}(f) \in M_{2 k}\left(N / 2, \chi_{0}^{2}\right)$ is a common eigen-function of $T_{\chi_{0}^{2}}(p)$, provided that $\mathscr{S}_{a, \chi_{0}}(f)$ is well-defined.

There is an obvious inclusion $M_{k+1 / 2}\left(N, \chi_{0}\right) \subset M_{k+1 / 2}\left(e N, \chi_{0}^{(e)}\right)$. So element $f$ of $M_{k+1 / 2}\left(N, \chi_{0}\right)$ has another lifting $\mathscr{S}_{a, \chi_{0}^{(c)}}(f)$. The relation between $\mathscr{S}_{a, \chi_{0}}$ and $\mathscr{S}_{a, \chi_{0}^{(e)}}$ is obtained by a formal computation as follows;

LeMma 5. Let $p_{1}, \ldots, p_{s}$ be all the prime factors of $e$. Then we have 


$$
\begin{aligned}
\mathscr{S}_{a, \chi_{0}^{(e)}}= & \left\{1-\sum_{i=1}^{s} \chi_{a^{*}}\left(p_{i}\right) \chi_{0}\left(p_{i}\right) p_{i}^{k-1} B_{p_{i}}+\sum_{1 \leq i, j \leq s} \chi_{a^{*}}\left(p_{i} p_{j}\right) \chi_{0}\left(p_{i} p_{j}\right)\left(p_{i} p_{j}\right)^{k-1} B_{p_{i} p_{j}}\right. \\
& \left.+\cdots+(-1)^{s} \chi_{a^{*}}\left(p_{1} \cdots p_{s}\right) \chi_{0}\left(p_{1} \cdots p_{s}\right)\left(p_{1} \cdots p_{s}\right)^{k-1} B_{p_{1} \cdots p_{s}}\right\} \circ \mathscr{S}_{a, \chi_{0}}
\end{aligned}
$$

provided that $\mathscr{S}_{a, \chi_{0}}$ is well-defined.

LEMMA 6. Let $4 \mid N, \chi_{0} \in(Z / N)^{*}$. Let a,e $\in N$ where a is square-free. Let $a / e=a_{0} / e_{0}^{2}$ with $a_{0}$ square-free. Then

$$
\mathscr{S}_{a, \chi_{0}^{\prime}} \circ B_{e}=B_{e_{0}} \circ \mathscr{S}_{a_{0}, \chi_{0}^{(e)}}
$$

with $\chi_{0}^{\prime}=\chi_{0}(e /|\cdot|)$, provided that $\mathscr{S}_{a_{0}, \chi_{0}^{(e)}}$ is well-defined.

ProOF. Let $f(z)=\sum_{n=0}^{\infty} c_{n} e(n z) \in M_{k+1 / 2}\left(N, \chi_{0}\right)$. Then $B_{e}(f)$ is in $M_{k+1 / 2}\left(e N, \chi_{0}^{\prime}\right)$. We have

$$
\mathscr{S}_{a, \chi_{0}^{\prime}}\left(B_{e}(f)\right)(z)=C+\sum_{n=1}^{\infty} \sum_{d \mid n} \chi_{a^{*}}(d) \chi_{0}(d)\left(\frac{e}{|d|}\right) d^{k-1} c_{a_{0} n^{2} / e_{0}^{2} d^{2}} \boldsymbol{e}(n z)
$$

Since $N$ is divisible by 4 and $\chi_{0}$ is a character $(\bmod N), \chi_{0}(d)$ and $\chi_{0}^{\left(e_{0}\right)}(d)$ vanish for $d$ even. It is easy to see that the equality $\chi_{a^{*}}(d) \chi_{0}(d)(e /|d|)=\chi_{a_{0}^{*}}(d) \chi_{0}^{(e)}(d)$ holds for $d$ odd. Then

$$
\begin{aligned}
\mathscr{S}_{a, \chi_{0}^{\prime}}\left(B_{e}(f)\right)(z) & =C+\sum_{n=1}^{\infty} \sum_{d \mid n} \chi_{a_{0}^{*}}(d) \chi_{0}^{(e)}(d) d^{k-1} c_{a_{0} n^{2} / d^{2}} \boldsymbol{e}\left(e_{0} n z\right) \\
& =B_{e_{0}}\left(\mathscr{S}_{a_{0}, \chi_{0}^{(e)}}(f)\right)(z) .
\end{aligned}
$$

Corollary. Let $\left\{f_{i, \chi_{0}}(z)\right\}_{i \in I\left(\chi_{0}\right)}$ be elements in $M_{k+1 / 2}\left(N, \chi_{0}\right)$ for $\chi_{0} \in$ $(Z / N)^{*}$. Let $M$ be the subspace of $M_{k+1 / 2}\left(\Gamma_{1}(N)\right)$ generated by $S_{k+1 / 2}(N)$ and by elements in the form $f_{i, \chi_{0}}(e z) \in M_{k+1 / 2}\left(\Gamma_{1}(N)\right)$ with $e \mid N, i \in I\left(\chi_{0}\right), \chi_{0} \in(Z / N)^{*}$. Suppose that $\mathscr{S}_{a, \chi_{0}}\left(f_{i, \chi_{0}}\right)$ are well-defined for all square-free a and for all $f_{i, \chi_{0}}, \chi_{0} \in$ $(Z / N)^{*}$. Then all the lifting maps $\mathscr{S}_{a, \chi_{0}}$ are well-defined on $M \cap M_{k+1 / 2}\left(N, \chi_{0}\right)$.

Proof. Since $M$ is spanned by $S_{k+1 / 2}(N)$ and by elements $f_{i, \chi_{0}}(e z)$, it is enough to show that $B_{e}\left(f_{i, \chi_{0}}\right) \in M_{k+1 / 2}\left(N, \chi_{0}(e /|\cdot|)\right)$ has liftings associated with all square-free $a$. Combining Lemma 5 with Lemma 6 , we see that they exist if 
$\mathscr{S}_{a, \chi_{0}}\left(f_{i, \chi_{0}}\right)$ exist for all $a$ and all $\chi_{0} \in(Z / N)^{*}$, which is our assumption. Then our assertion follows.

q.e.d.

5. In our previous paper [8, Prop. 4 , Lemma 5, Cor. to Theorem 2], we have shown the following;

THEOREM ([8]). Let $k \in \boldsymbol{N}$. Let $N=N_{1} N_{2} \in \boldsymbol{N}, \chi^{\prime} \in\left(Z / N_{1}\right)^{*}, \chi \in\left(Z / N_{2}\right)^{*}$, $\chi_{0}:=\chi \chi^{\prime} \in(Z / N)^{*}$ where $N$ is not necessarily divisible by 4 and $\chi$ is not necessary primitive. Suppose that a character $\chi_{0} \in(Z / N)^{*}$ has the same parity as $k$. Let $a \in N$ be square-free. Put $l=2^{\sharp\left\{i \mid v_{2}\left(\left(a^{*}, N_{i}\right)\right) \geq 2\right\}}$. Then

$$
\lambda_{2 k, a^{*}, \chi}^{\chi^{\prime}}(z):=C+4 \sum_{n=1}^{\infty} \sum_{0<d \mid n} \chi_{a^{*}}(d) \chi_{0}(d) d^{k-1} \sum_{m \in Z} \sigma_{k-1, \chi}^{\chi^{\prime}}\left(\frac{(n / d)^{2} a^{*}-m^{2}}{4}\right) \boldsymbol{e}(n z)
$$

with a suitable constant $C$, is a modular form in $M_{2 k}\left(N / l, \chi_{0}^{2}\right)$. If $k>1$, then $C=$ $L(1-k, \chi) L\left(1-k, \chi \chi_{a^{*}}\right)\left(N_{1}=1\right)$ and $C=0\left(N_{1}>1\right)$.

To construct the modular form in the theorem, we have made use of Hilbert Eisenstein series of real quadratic fields. We put $\lambda_{2 k, a^{*}, \chi}(z):=\lambda_{2 k, a^{*}, \chi}^{\chi^{\prime}}(z)\left(N_{1}=1\right)$, $\lambda_{2 k, a^{*}}^{\chi^{\prime}}(z):=\lambda_{2 k, a^{*}, \chi}^{\chi^{\prime}}(z)\left(N_{2}=1\right)$.

LemMA 7. Let $a, N, N_{1}, N_{2}, \chi^{\prime} \in\left(Z / N_{1}\right)^{*}, \chi \in\left(Z / N_{2}\right)^{*}, \chi_{0} \in(Z / N)^{*}$ be as in the above theorem.

(1) Suppose that $G_{k, \chi}^{\chi^{\prime}}$ is holomorphic.

(i) Let $a \equiv 1(\bmod 4)$. Then

$$
\begin{gathered}
\mathscr{S}_{a, \chi_{0}}\left(\theta(z) G_{k, \chi}^{\chi^{\prime}}(z)\right)=U_{2}\left(\lambda_{2 k, a^{*}, \chi}^{\chi^{\prime}}(z)\right), \\
\mathscr{S}_{a, \chi_{0}}\left(\theta(z) G_{k, \chi}^{\chi^{\prime}}(4 z)\right)=\lambda_{2 k, a^{*}, \chi}^{\chi^{\prime}}(z), \\
\mathscr{S}_{a, \chi_{0}}\left(\theta(z) G_{k, \chi}^{\chi^{\prime}}(2 z)\right)=2^{k-1} \chi(2) \lambda_{2 k, a^{*}, \chi}^{\chi^{\prime}}(z) \quad\left(v_{2}\left(N_{1}\right)>0\right), \\
\mathscr{S}_{a, \chi_{0}}\left(\theta(z) G_{k, \chi}^{\chi^{\prime}}(2 z)\right)=2^{k-1} \chi^{\prime}(2) \lambda_{2 k, a^{*}, \chi}^{\chi^{\prime}}(z) \quad\left(v_{2}\left(N_{2}\right)>0\right),
\end{gathered}
$$

which are modular forms in $M_{2 k}\left(N, \chi_{0}^{2}\right)$ where the first one is in $M_{2 k}\left(N / 2, \chi_{0}^{2}\right)$ if $v_{2}(N) \geq 2$.

(ii) Let $a \not \equiv 1(\bmod 4)$. Then

$$
\begin{gathered}
\mathscr{S}_{a, \chi_{0}}\left(\theta(z) G_{k, \chi}^{\chi^{\prime}}(z)\right)=\lambda_{2 k, a^{*}, \chi}^{\chi^{\prime}}(z), \\
\mathscr{S}_{a, \chi_{0}}\left(\theta(z) G_{k, \chi}^{\chi^{\prime}}(4 z)\right)=B_{2}\left(\lambda_{2 k, a^{*}, \chi}^{\chi^{\prime}}(z)\right)
\end{gathered}
$$


where the former is in $M_{2 k}\left(N / l, \chi_{0}^{2}\right)$ and the latter in $M_{2 k}\left(2 N / l, \chi_{0}^{2}\right)$ with $l=$

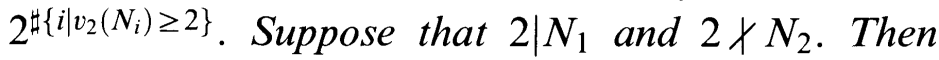

$$
\mathscr{S}_{a, \chi_{0}}\left(\theta(z) G_{k, \chi}^{\chi^{\prime}}(2 z)\right)=2^{-k+1} \chi(2)^{-1}\left(\lambda_{2 k, a^{*}, \chi}^{\chi^{\prime}}(z)-\lambda_{2 k, a^{*}, \chi^{(2)}}^{\chi^{\prime}}(z)\right),
$$

which is in $M_{2 k}\left(2 N / l, \chi_{0}^{2}\right)$ with $l=1 \quad\left(v_{2}\left(N_{1}\right) \leq 1\right), 2$ (otherwise).

(2) If $b$ denotes 1 or 2 according as $N_{1}$ is odd or even, then a function $b G_{2, \chi}^{\chi^{\prime}}(2 z)-G_{2, \chi}^{\chi^{\prime}(2)}(z)$ is holomorphic even when $k=2$. Let $N_{2}$ be odd. Then $\mathscr{S}_{a, \chi_{0}}\left(\theta(z)\left(b G_{2, \chi}^{\chi^{\prime}}(4 z)-G_{2, \chi}^{\chi^{\prime}(2)}(2 z)\right)\right.$ is equal to

$$
\begin{gathered}
b \lambda_{2 k, a^{*}, \chi}^{\chi^{\prime}}(z)-2^{k-1} \chi(2) \lambda_{2 k, a^{*}, \chi}^{\chi^{\prime}(2)}(z) \quad(a \equiv 1(\bmod 4)), \\
b B_{2}\left(\lambda_{2 k, a^{*}, \chi}^{\chi^{\prime}}(z)\right)-2^{-k+1} \chi(2)^{-1}\left(\lambda_{2 k, a^{*}, \chi}^{\chi^{\prime}(2)}(z)-\lambda_{2 k, a^{*}, \chi^{(2)}}^{\chi^{\prime}(2)}(z)\right) \quad(a \neq 1(\bmod 4)),
\end{gathered}
$$

which is in $M_{2 k}\left(2 N, \chi_{0}^{2}\right)(a \equiv 1(\bmod 4))$, or in $M_{2 k}\left(4 N / b, \chi_{0}^{2}\right)(a \not \equiv 1(\bmod 4))$. Let $2 \mid N_{1}$. Then $\mathscr{S}_{a, \chi_{0}}\left(\theta(z)\left(2 G_{2, \chi}^{\chi^{\prime}}(2 z)-G_{2, \chi}^{\chi^{\prime}(2)}(z)\right)\right.$ is equal to

$$
\begin{gathered}
2^{k} \chi(2) \lambda_{2 k, a^{*}, \chi}^{\chi^{\prime}}(z)-U_{2}\left(\lambda_{2 k, a^{*}, \chi}^{\chi^{\prime}}(z)\right) \quad(a \equiv 1(\bmod 4)), \\
2^{-k+2} \chi(2)^{-1}\left(\lambda_{2 k, a^{*}, \chi}^{\chi^{\prime}}(z)-\lambda_{2 k, a^{*}, \chi^{(2)}}^{\chi^{\prime}}(z)\right)-\lambda_{2 k, a^{*}, \chi}^{\chi^{\prime}}(z) \quad(a \neq \equiv 1(\bmod 4)),
\end{gathered}
$$

which is in $M_{2 k}\left(N, \chi_{0}^{2}\right)(a \equiv 1(\bmod 4))$, or in $M_{2 k}\left(2 N / l, \chi_{0}^{2}\right)(a \not \equiv 1(\bmod 4))$ with $l=1\left(v_{2}\left(N_{1}\right) \leq 1\right), 2$ (otherwise).

Proof. The first and second equalities in (i) of (1) are easily obtained. We prove the third one. We have

$$
\mathscr{S}_{a, \chi_{0}}\left(\theta(z) G_{k, \chi}^{\chi^{\prime}}(2 z)\right)=C+\sum_{n=1}^{\infty} \sum_{0<d \mid n} \chi_{a}(d) \chi_{0}(d) d^{k-1} \sigma_{k-1, \chi}^{\chi^{\prime}}\left(\frac{a(n / d)^{2}-m^{2}}{2}\right) \boldsymbol{e}(n z) .
$$

Since $a \equiv 1(\bmod 4),\left(a(n / d)^{2}-m^{2}\right) / 2$ is even whenever it is integral. Then $\sigma_{k-1, \chi}^{\chi^{\prime}}\left(\left(a(n / d)^{2}-m^{2}\right) / 2\right)=2^{k-1} \chi(2) \sigma_{k-1, \chi}^{\chi^{\prime}}\left(\left(a(n / d)^{2}-m^{2}\right) / 4\right)$, and hence the equality follows. The fourth one is proved similarly.

The first and second equations in (ii) of (1) is again immediate. We prove the third one. Note that $\chi_{0}=\chi^{\prime} \chi=\chi^{\prime} \chi^{(2)}$. Then

$$
\begin{aligned}
& \lambda_{2 k, a^{*}, \chi}^{\chi^{\prime}}(z)-\lambda_{2 k, a^{*}, \chi^{(2)}}^{\chi^{\prime}}(z) \\
& \quad=C+4 \sum_{n=1}^{\infty} \sum_{0<d \mid n} \chi_{4 a}(d) \chi_{0}(d) d^{k-1} \sum_{\substack{m \in Z \\
2 \mid\left(a(n / d)^{2}-m^{2}\right)}} \sigma_{k-1, \chi}^{\chi^{\prime}}\left(a(n / d)^{2}-m^{2}\right) \boldsymbol{e}(n z)
\end{aligned}
$$




$$
\begin{aligned}
& =C+4 \cdot 2^{k-1} \chi(2) \sum_{n=1}^{\infty} \sum_{0<d \mid n} \chi_{4 a}(d) \chi_{0}(d) d^{k-1} \sum_{m \in Z} \sigma_{k-1, \chi}^{\chi^{\prime}}\left(\frac{a(n / d)^{2}-m^{2}}{2}\right) e(n z) \\
& =2^{k-1} \chi(2) \mathscr{S}_{a, \chi_{0}}\left(\theta(z) G_{k, \chi}^{\chi^{\prime}}(2 z)\right) .
\end{aligned}
$$

The equalities in (2) follow from the computations in (1).

q.e.d.

THEOREM 1. Let $k \geq 1,4 \mid N, \chi_{0} \in(Z / N)^{*}$. Let $a \in N$ be square-free. Then the Shimura lifting map $\mathscr{S}_{a, \chi_{0}}$ is a Hecke equivariant linear map of $G_{k+1 / 2}\left(N, \chi_{0}\right) \oplus$ $S_{k+1 / 2}\left(N, \chi_{0}\right)$ to $M_{2 k}\left(N / 2, \chi_{0}^{2}\right)$.

Proof. Let $k \neq 2$. By Lemma 7, $\theta(z) G_{k, \chi}^{\chi^{\prime}}(z)\left(N=N_{1} N_{2}, e\left|N_{2}, 4\right| N_{1}\right.$ or $\left.4 \mid\left(N_{2} / e\right), \chi^{\prime} \in\left(Z / N_{1}\right)^{*}, \chi \in\left(Z /\left(N_{2} / e\right)\right)^{*}\right) \quad$ and $\theta(z) G_{k, \chi}^{\chi^{\prime}}(4 z)\left(N=N_{1} N_{2}, 4|e| N_{2}\right.$, $\left.\chi^{\prime} \in\left(Z / N_{1}\right)^{*}, \chi \in\left(Z /\left(N_{2} / e\right)\right)^{*}\right)$ have liftings in $M_{2 k}\left(N / 2 e,\left(\chi \chi^{\prime}\right)^{2}\right)$ and in $M_{2 k}\left(N / e,\left(\chi \chi^{\prime}\right)^{2}\right)$ respectively for any square-free $a$. We can take $G_{k+1 / 2}\left(N, \chi_{0}\right) \oplus$ $S_{k+1 / 2}\left(N, \chi_{0}\right)$, as $M$ in Corollary to Lemma 6. Hence the lifting map is welldefined on $G_{k+1 / 2}\left(N, \chi_{0}\right) \oplus S_{k+1 / 2}\left(N, \chi_{0}\right)$. By Lemma 6, it is easy to see that the image is in $M_{2 k}\left(N / 2, \chi_{0}^{2}\right)$. This shows our assertion.

Let $k=2$. Then we must take into account modular forms $\theta(z) \times$ $\left(b G_{2}^{1_{N_{1}}}(4 z)-G_{2}^{1_{2 N_{1}}}(2 z)\right), \theta(z)\left(2 G_{2}^{1_{\left(N_{1} / 2\right)}}(2 z)-G_{2}^{1_{N_{1}}}(z)\right)$ in Proposition 3 (2). To this case we can apply Lemma 7 (2) and our assertion follows in the same manner as in the case $k \neq 2$.

q.e.d.

From Corollary to Proposition 3, we obtain the main theorem of the paper.

THEOREM 2. Let $k \geq 2$. Let $a, \chi_{0}$ be as in Theorem 1. Then the Shimura lifting map $\mathscr{S}_{a, \chi_{0}}$ is a Hecke equivariant linear map of $M_{k+1 / 2}\left(N, \chi_{0}\right)$ to $M_{2 k}\left(N / 2, \chi_{0}^{2}\right)$.

REMARK. Let $k \geq 2$. Let $E_{k+1 / 2}\left(N, \chi_{0}\right)$ denote the orthogonal complement of $S_{k+1 / 2}\left(N, \chi_{0}\right)$ in $M_{k+1 / 2}\left(N, \chi_{0}\right)$ with respect to the Petersson product. Then the space $M_{k+1 / 2}\left(N, \chi_{0}\right)$ is decomposed into $E_{k+1 / 2}\left(N, \chi_{0}\right) \oplus S_{k+1 / 2}\left(N, \chi_{0}\right)$ as Hecke modules. An eigen-function of all Hecke operators in $E_{k+1 / 2}\left(N, \chi_{0}\right)$ is mapped to an eigen-function in $M_{2 k}\left(N, \chi_{0}^{2}\right)$. By the growth condition of Fourier coefficients, it is shown that the eigen-function in $M_{2 k}\left(N, \chi_{0}^{2}\right)$ is in $E_{2 k}\left(N, \chi_{0}^{2}\right)$. Hence the lifting map sends Eisenstein series of half-integral weight to Eisenstein series of integral weight.

The case $k=1$ of Theorem 2 will be investigated in our later paper. 
6. We give two applications of our theorem.

(1) Let $S$ be a positive definite symmetric integral matrix of size $2 k+1$. Let $N$ be a multiple of 4 such that $1 /(4 N) S^{-1}$ is integral. Let

$$
\theta_{S}(z):=\sum_{g \in \boldsymbol{Z}^{2 k+1}} \boldsymbol{e}\left({ }^{t} g S g z\right)
$$

Then $\theta_{S}(z) \in M_{k+1 / 2}\left(\Gamma_{0}(N), \chi_{-4}^{k} \cdot(\operatorname{det}(S) /)\right)$. Let $r_{s}(n), n \in N$, denote the number of integral solutions of an equation ${ }^{t} X S X=n$ where $X$ is a variable vector with $2 k+1$ elements. We have

$$
\theta_{S}(z)=1+\sum_{n=1}^{\infty} r_{S}(n) \boldsymbol{e}(n z)
$$

By Theorem 2, the lifting of this modular form is in $M_{2 k}\left(N / 2,1_{N / 2}\right)$. We state this as a proposition.

Proposition 4. Let $S, N$ be as above with $k \geq 2$. Let a be any square-free natural number. Let $a_{0} a_{1}^{2}=(-4)^{k} a \cdot \operatorname{det}(S)$ where $a_{0}$ is 1 or the discriminant of a quadratic field. Then for a suitable constant $C$,

$$
C+\sum_{n=1}^{\infty}\left(\sum_{0<d \mid n} \chi_{a_{0}}^{\left(a_{1}\right)}(d) d^{k-1} r_{S}\left(a(n / d)^{2}\right)\right) e(n z)
$$

is a modular form in $M_{2 k}\left(N / 2,1_{N / 2}\right)$.

In case that $S$ has an odd prime determinant, the result was first obtained by van Asch [1, Theorem 2], where the constant term $C$ is written explicitly in terms of $S$ and $a$.

(2) We derive several relations among arithmetic functions or special values of $L$-functions.

(i) Since $j(M, z)^{2}=\chi_{-4}(d)(c z+d)$ for $M=\left(\begin{array}{ll}a & b \\ c & d\end{array}\right) \in \Gamma_{0}(4)$, we have an inclusion $\theta(z) M_{7 / 2}\left(4, \chi_{-4}\right) \subset M_{4}(4)$. This shows in particular, that $S_{7 / 2}\left(4, \chi_{-4}\right)=$ $\{0\}$ because $S_{4}(4)=\{0\}$. Thus $\operatorname{dim}_{C} M_{7 / 2}\left(4, \chi_{-4}\right)$ is at most 2 , the number of regular cusps. The modular forms $31 \theta(z) G_{3, \chi_{-4}}(z)-16 \theta(z) G_{3}^{\chi_{-4}}(z), \theta(z) G_{3}^{\chi_{-4}}(z)$ spans $M_{7 / 2}\left(4, \chi_{-4}\right)$. Hence $M_{7 / 2}\left(4, \chi_{-4}\right)=G_{7 / 2}\left(4, \chi_{-4}\right)$, and the space is of dimension two. It is easy to see only be applying $U_{4}$, that they are common Hecke eigen-functions. The eigenvalue of $\theta(z) G_{3}^{\chi-4}(z)$ for $U_{4}$ is $2^{5}$. Since $G_{6}(z)-G_{6}(2 z)$ is the unique function in $M_{2}\left(2,1_{2}\right)$, up to constant factor, such that 
$U_{2}\left(G_{6}(z)-G_{6}(2 z)\right)=2^{5}\left(G_{6}(z)-G_{6}(2 z)\right)$, we have

$$
\mathscr{S}_{a^{*}, \chi-4}\left(\theta(z) G_{3}^{\chi-4}(z)\right)=\sum_{m \in \boldsymbol{Z}} \sigma_{2}^{\chi-4}\left(a-m^{2}\right)\left(G_{6}(z)-G_{6}(2 z)\right)
$$

by comparing the first Fourier coefficients. Then comparing higher terms, we obtain

$$
\sum_{m \in \boldsymbol{Z}} \sigma_{2}^{\chi-4}\left(a-m^{2}\right) \sigma_{5}^{1_{2}}(n)-\sum_{\substack{0<d \mid n \\ d: o d d}} \chi^{\prime}(d) d^{2} \sum_{m \in \boldsymbol{Z}} \sigma_{2}^{\chi-4}\left((n / d)^{2} a-m^{2}\right)=0
$$

for any $n \in N$, where $\chi^{\prime}=\chi_{-4 a}(a \equiv 1,2(\bmod 4)), \chi_{-a}$ (otherwise).

(ii) Let $k \geq 4$. The Shimura lifting $\mathscr{S}_{a, 1_{4}}\left(\theta(z) G_{k}(4 z)\right)(a \equiv 1(\bmod 4))$ or $\mathscr{S}_{4 a, 1_{4}}\left(\theta(z) G_{k}(z)\right)(a \equiv 2,3(\bmod 4))$, namely, $\lambda_{2 k, a^{*}, 1}$ is in $M_{2 k}(1)$. Let $k=4$. Then $M_{8}(1)$ is of dimension one, and hence

$$
\lambda_{8, a^{*}, 1}(z)=\zeta(-3) L\left(-3, \chi_{a^{*}}\right)+4 \sum_{n=1}^{\infty} \sum_{0<d \mid n} \chi_{a^{*}}(d) d^{3} \sum_{m \in \boldsymbol{Z}} \sigma_{3}\left(\frac{(n / d)^{2} a^{*}-m^{2}}{4}\right) \boldsymbol{e}(n z)
$$

is equal to

$$
G_{8}(z)=\zeta(-7)+2 \sum_{n=1}^{\infty} \sigma_{7}(n) \boldsymbol{e}(n z)
$$

up to a constant multiple. Then we have an identity

$$
L\left(-3, \chi_{a^{*}}\right) \sigma_{7}(n)-\sum_{0<d \mid n} \chi_{a^{*}}(d) d^{3} \sum_{m \in Z} \sigma_{3}\left(\frac{(n / d)^{2} a^{*}-m^{2}}{4}\right)=0
$$

for any $a \in N$, square-free. When $a=1$, this gives the well-known formula

$$
\sigma_{7}(n)-120 \sum_{m=0}^{n} \sigma_{3}(m) \sigma_{3}(n-m)=0
$$

because $L\left(-3, \chi_{1}\right)=\zeta(-3)=1 / 120, \quad \sigma_{3}(0)=1 / 2 \zeta(-3)=1 / 240$ and $\sum_{0<d \mid n} d^{3}$. $\sum_{m \in Z} \sigma_{3}\left(\frac{(n / d)^{2}-m^{2}}{4}\right)=\sum_{m=0}^{n} \sigma_{3}(n) \sigma_{3}(n-m)$ (for example, see [8], the section 3).

(iii) Let us consider the case $k=6$ in (ii). Two modular forms

$$
\begin{gathered}
\lambda_{12, a^{*}, 1}(z)=-\frac{1}{252} L\left(-5, \chi_{a^{*}}\right)+4 \sum_{n=1}^{\infty} \sum_{0<d \mid n} \chi_{a^{*}}(d) d^{5} \sum_{m \in \boldsymbol{Z}} \sigma_{5}\left(\frac{(n / d)^{2} a^{*}-m^{2}}{4}\right) \boldsymbol{e}(n z), \\
G_{12}(z)=\frac{691}{32760}+2 \sum_{n=1}^{\infty} \sigma_{11}(n) \boldsymbol{e}(n z)
\end{gathered}
$$


are in $M_{12}(1)$. Let $\tau$ denote the Ramanujan function, namely $\Delta(z)=$ $\sum_{n=1}^{\infty} \tau(n) \boldsymbol{e}(n z), \tau(1)=1$, where $\Delta(z)$ denotes the cusp form in $M_{12}(1)$. Then $-1 / 252 L\left(-5, \chi_{a^{*}}\right) G_{12}(z)-691 / 32760 \lambda_{12, a^{*}}(z)=-1 / 126\left\{L\left(-5, \chi_{a^{*}}\right)+\right.$ $\left.691 / 65 \sum_{m \in Z} \sigma_{5}\left(\left(a^{*}-m^{2}\right) / 4\right)\right\} \Delta(z)$, and we have

$$
\begin{aligned}
& \left\{65 L\left(-5, \chi_{a^{*}}\right)+691 \sum_{m \in Z} \sigma_{5}\left(\frac{a^{*}-m^{2}}{4}\right)\right\} \tau(n) \\
& \quad=65 L\left(-5, \chi_{a^{*}}\right) \sigma_{11}(n)+691 \sum_{0<d \mid n} \chi_{a^{*}}(d) d^{5} \sum_{m \in Z} \sigma_{5}\left(\frac{(n / d)^{2} a^{*}-m^{2}}{4}\right) .
\end{aligned}
$$

\section{References}

[1] A. G. Van Asch, Modular forms of half integral weight, some explicit arithmetic, Math. Ann., 262 (1983), 77-89.

[2] E. Hecke, Theorie der Eisenstein Reihen höhere Stufe und ihre Anwendung auf Funktionentheorie und Arithmetik, Abh. Math. Sem. Univ. Hamburg, 5 (1927), 199-224.

[ 3 ] W. Kohnen and D. Zagier, Values of $L$-series of modular forms at the center of the critical strip, Invent. Math., 64 (1981), 175-198.

[4] S. Niwa, Modular forms of half-integral weight and the integral of certain theta-functions, Nagoya Math. J. 56 (1974), 147-161.

[ 5 ] D. Pei, A basis of Eisenstein series of half-integral weight, SEA Bull. Math., 15 (1991), 49-55.

[6] G. Shimura, Modular forms of half integral weight; In Modular functions of one variable, Lect. Notes in Math., 320, 59-74, Springer-Verlag, Berlin, Heidelberg, New York, 1972.

[ 7 ] G. Shimura, On modular forms of half integral weight, Ann. Math., 97 (1973), 440-481.

[ 8 ] S. Tsuyumine, On values of $L$-functions of totally real algebraic number fields at integers, Acta Arith., 76 (1996), 359-392.

[ 9 ] D. Zagier, Modular forms associated to real quadratic fields, Invent. Math., 30 (1975), 1-46.

Department of Mathematics

Mie University

Tsu, 514-8507, Japan 